\title{
Decision making under conflict: Decision time as a measure of conflict strength
}

\author{
ADELE DIEDERICH \\ International University Bremen, Bremen, Germany
}

\begin{abstract}
Conflict and choice are closely related in that choice produces conflict and conflict is resolved by making a choice. Although conflict was invoked in psychological approaches to decision making early on (Lewin, 1931/1964), no generally accepted measure of conflict strength has been established (Tversky \& Shafir, 1992). The present study introduces a model (multiattribute decision field theory) that predicts a decision time pattern depending on the conflict situation. In a risky decision-making experiment with multiattribute choice alternatives, decision time is investigated as a possible measure of conflict strength. It is shown that the model can be fitted to a complex choice pattern.
\end{abstract}

Everyday experience suggests that decision making is often accompanied by conflict. For example, both farreaching decisions-for instance, whether to have chemotherapy or surgery as a treatment for cancer-and trifling decisions, of whether to go to a concert or an opera, can generate conflict. A conflict is resolved by making a choice between alternatives. Kurt Lewin was among the first to bring together the concepts of decision making and psychological conflict (Lewin, 1951, 1931/1964). Depending on the valences of the choice alternatives, conflict situations emerge that have been classified as approach-approach conflict situations, avoidanceavoidance conflict situations, or approach-avoidance conflict situations (Miller, 1944). In an approachapproach conflict situation, a decision is made between desirable alternatives; in an avoidance-avoidance conflict situation, the choice alternatives are undesirable; in an approach-avoidance conflict situation, the choice alternatives possess both desirable and undesirable features. Furthermore, the uncertainty about possible consequences of a decision may intensify conflict (see, e.g., Hogarth, 1975).

Several approaches that invoke conflict situations in the analysis of decision making have been proposed (e.g., Busemeyer \& Townsend, 1993; Coombs \& Avrunin, 1988; Diederich, 1997; Hull, 1932; Janis \& Mann, 1977; Lewin, 1931/1964; Mellers, Schwartz, Ho, \& Ritov, 1997; Miller, 1944; Townsend \& Busemeyer, 1989), but as Tversky and Shafir (1992) pointed out, there exists neither a standard definition of conflict nor a generally accepted procedure for measuring conflict. Assuming that decision making in conflict situations is more difficult than in no-

This research was supported by Deutsche Forschungsgemeinschaft Grants Di 506/5-1 and Di 506/6-1. Correspondence concerning this article should be addressed to A. Diederich, School of Humanities and Social Sciences, International University Bremen, P. O. Box 750561 , D-28725 Bremen, Germany (e-mail: a.diederich@iu-bremen.de). conflict situations, they suggested the deferring of a decision as an indicator of conflict. Further experimental findings indicate that it takes longer to reach a decision in avoidance-avoidance conflict situations than in approachapproach conflict situations (e.g., Berlyne, 1957; Böckenholt, Albert, Aschenbrenner, \& Schmalhofer, 1991; Busemeyer, 1985; Dashiell, 1937; Hansen, 1972; Houston, Sherman, \& Baker, 1991; Janis \& Mann, 1977; Luce, Bettman, \& Payne, 1997).

The purpose of the present study was (1) to introduce a model that actually predicts a decision time pattern depending on conflict situation type and (2) to probe the assumption that decision time can be used as a measure of conflict strength in risky decision making. To test this idea, experimentally multiattribute choice alternatives were constructed. The advantage of multiattribute choice alternatives is that they may have desirable attributes, undesirable attributes, or both. Moreover, the level of desirability/undesirability may vary for each attribute. Finally, the variability of their values can be manipulated for each attribute, so as to test the assumption that uncertainty about the consequences may induce conflict. Conflict will be manipulated by varying the relative desirability of the choice alternatives. The resulting choice probability pattern will depend on the preference for particular attributes and will allow one to infer the type of conflict experienced by the participant. Thus, the choice probability pattern will be used to predict the ordered relations of decision time that presumably indicates the conflict strength.

In the following, I will briefly present a dynamic stochastic decision model for binary multiattribute decision problems, called the multiattribute decision field theory (MDFT), and its specific predictions for decision making in conflict situations. Details - in particular, the mathematical derivations-are given in Diederich (1996, 1997). MDFT is a sequential comparison model that extends and generalizes the so-called decision field theory (DFT) of Busemeyer and Townsend $(1992,1993)$ from 
Table 1

Four Medical Treatments (Choice Alternatives) With Three Attributes

\begin{tabular}{lllll}
\hline & \multicolumn{4}{c}{ Alternative } \\
\cline { 2 - 5 } \multicolumn{1}{c}{ Attribute } & A & B & C & D \\
\hline Cost & high & high & low & low \\
Intensity of pain & low & low & high & high \\
Variability of recovery time & low & high & low & high \\
\hline
\end{tabular}

unidimensional to multidimensional (i.e., multiattribute) choice alternatives. Then an experiment will be reported, and the model will be fitted to the data.

\section{MULTIATTRIBUTE DECISION FIELD THEORY}

To illustrate the model and its predictions, consider a very simple example. Assume that there are four medical treatments available with three attributes, as is shown in Table 1. The treatments differ with respect to cost and intensity of pain a patient usually has to endure. Furthermore, the mean time to recover is the same for all four treatments, but the variance of the recovery time differs. Assume that a hypothetical patient, the decision maker (DM), prefers C over A and D over B; then, we would infer that cost is more important than intensity of pain. Thus, we assume that choosing between $C$ and $D$ (both alternatives with low values for cost) evokes an approach conflict, ${ }^{1}$ whereas choosing between A and B (both alternatives with high cost) evokes an avoidance conflict. With respect to our hypothesis, we would expect that it would take longer to make a decision between $A$ and $B$ than between $C$ and D. Furthermore, assume that the DM prefers B over A and D over C; then, we would infer that high variability is preferred to low variability (risk seeking), and therefore, we assume that it evokes an approach conflict when choosing between B and $\mathrm{D}$ (both alternatives with high variability) and an avoidance conflict when choosing between $\mathrm{A}$ and $\mathrm{C}$ (both alternatives with low variability), other attribute levels being the same. According to the hypothesis, we would expect that it would take longer to make a decision between $\mathrm{A}$ and $\mathrm{C}$ than between $\mathrm{B}$ and $\mathrm{D}$. For the choice pairs $\mathrm{AD}$ and $\mathrm{BC}$, we cannot make any predictions on this qualitative level. However, MDFT, which will be introduced next, accounts for the entire pattern of data.

MDFT provides a formal description of the dynamic evolution of preference during deliberation for multiattribute choice alternatives. The main task is to predict which alternative will be chosen and the time it will take to make that choice.

Deliberation starts at time $t=0$, and at each point in time during deliberation, there is a strength of preference, denoted $P(t)$, for choosing one alternative over the other. For example, if a choice is to be made between $\mathrm{A}$ and $\mathrm{B}, P(t)$ would be the relative strength of preference of choosing A over B at time $t$. More specifically, a pos- itive value of $P(t)$ represents a preference strength in the direction of favoring $\mathrm{A}$, whereas a negative value of $P(t)$ represents a preference strength in the direction of favoring B. Preference strength is updated from one moment, $t$, to the next, $t+\tau$, by an input valence reflecting the momentary comparison of consequences produced by imaging the choice of either A or B. The valence fluctuates because the DM's attention switches back and forth between anticipated consequences.

For multiattribute alternatives, it is assumed that the preference process has a specific input valence $V_{i}(t)$ for each attribute comparison. The DM draws information about the alternatives and their attributes from his or her memory. The possible consequences connected with either alternative are learned from experience and are remembered more or less well. A decision is made as soon as the preference process reaches a preset decision criterion, or threshold, for either of the two alternatives (see Diederich \& Busemeyer, 1999). The dynamics of the preference process is formally described according to the following linear dynamic model ( $\tau$ is a small time unit):

$$
P(t+\tau)=\left(1-\tau \cdot \gamma_{i}\right) P(t)+V_{i}(t+\tau) .
$$

The subscript $i$ refers to the attribute considered at time $t$, and $\gamma_{i}$ determines the growth or decay of the preference process with respect to attribute $i$ and is related to the distinction between approach and avoidance conflicts. For $\gamma>0$, the preference process decays over time, and thus, it takes longer to reach the decision criterion. This describes avoidance-avoidance conflicts. The increments of preference become smaller and smaller, the closer the process is to the decision boundary. It slows down the process, and therefore, it prolongs the time before a decision is made. For $\gamma<0$, the preference process accelerates over time, and it takes less time to reach the decision criterion. This describes approach-approach conflicts. The increments of preferences become larger and larger, the closer the process is to the decision criterion. For $\gamma=0$, no conflict exists, and the preference process grows linearly over time. The mean valence for each attribute comparison is $E\left[V_{i}(t)\right]=\delta_{i} t$, where $\delta_{i}$, called the drift rate, indicates the direction toward choosing A or $\mathrm{B}$. In particular, for $\delta_{i}>0$, mean preference is directed toward $\mathrm{A}$, whereas for $\delta_{i}<0$, it is directed toward $\mathrm{B}$. The process switches (attention shift) from attribute $i$ to attribute $j$ at a particular rate $w_{i j}$. That is, attention switches according to a mixture of two subprocesses, $W_{1}(t)$ and $W_{2}(t)$. At any particular time during deliberation, the attention process may be operating on the basis of one of these subprocesses-say $W_{1}(t)$. During the next moment, attention either continues to operate under process $W_{1}(t)$ with a probability of $w_{11}$ or attention switches with a probability of $w_{12}=1-w_{11}$ and starts operating on the basis of $W_{2}(t)$. Similarly, if attention is operating on the basis of $W_{2}(t)$, then during the next moment, attention may continue under $W_{2}(t)$ with a probability of $w_{22}$, or may switch to $W_{1}(t)$ with a probability of $w_{21}=1-w_{22}$. 


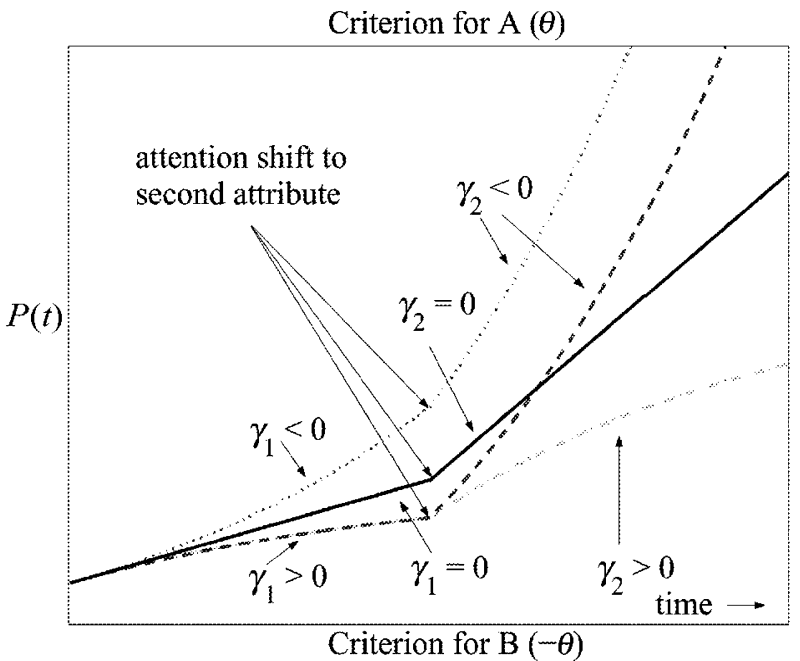

Figure 1. Mean preference process $P(t)$ over time with a mean valence toward $\mathrm{A}\left(\delta_{1}>\mathbf{0}, \delta_{2}>0\right) . \gamma_{1}<0$ indicates an approach conflict when considering Attribute $1\left(\ldots\right.$ line); $\gamma_{1}>0$ indicates an avoidance conflict when considering Attribute 1 ( -- line; - - line); $\gamma_{2}=0$ indicates no conflict (solid line). $\gamma_{2}<0$ indicates an approach conflict when considering Attribute 2 ( $\cdots$ line; -- line); $\gamma_{2}>0$ indicates an avoidance conflict when considering Attribute 2 (- - line).

Thus, attention switches from one attribute to another according to a Markov chain process. ${ }^{2}$ Roe, Busemeyer, and Townsend (2001) also included the notion of multiattribute alternatives in another extended version of DFT, also called MDFT. ${ }^{3}$ The basic difference between their approach and the model represented here is that, in the former, the drift rate is a weighted average of the attributes, whereas in the latter, attention switches according to a mixture of the subprocesses. In a sense, one could say that Roe et al.'s MDFT assumes that attributes are processed in parallel, whereas in the approach presented here, the attributes are processed in a serial manner. For details, see Busemeyer and Diederich (2002).

The preference process stops and a decision is initiated as soon as the process reaches a decision criterion, $\theta>0$. If $P(t)>\theta$, A is chosen; if $-P(t)>\theta$, B is chosen. The criterion is assumed to be set by the DM prior to the decision task. Therefore, the probability of choosing A over B is determined by $P(t)$ 's reaching the positive threshold before reaching the negative threshold. Furthermore, the decision time is defined as the time from the start of the trial until $P(t)$ reaches a threshold bound. Figure 1 illustrates processes for different conflict situations. For simplicity's sake, choice alternatives have only two attributes. In this example, the mean valences, $\delta_{1}=.1$ and $\delta_{2}=.3$, are directed toward choosing $\mathrm{A}$. The lines indicates the mean preference. That is, the dotted line represents a pure approach conflict $\left(\gamma_{1}, \gamma_{2}<0\right)$; the dash-point line represents a pure avoidance conflict $\left(\gamma_{1}\right.$, $\left.\gamma_{2}>0\right)$; the dash line represents an approach-avoidance conflict - that is, an avoidance conflict when consider- ing Attribute $1\left(\gamma_{1}>0\right)$ and an approach conflict when considering Attribute $2\left(\gamma_{2}<0\right)$. When $\gamma=0$ (solid line), no conflict is assumed.

MDFT quantitatively predicts which alternative will be chosen and the time it takes to make that choice. To demonstrate this, consider again the example above. A value of one was assigned to attribute level low, a value of zero to attribute level high. Comparison of two alternatives with respect to an attribute results in a comparison value that is simply taken as the difference between these values. For example, comparing alternatives $\mathrm{A}$ and $\mathrm{C}$ with respect to cost gives the comparison value -1 ; comparing $\mathrm{A}$ and $\mathrm{C}$ with respect to intensity of pain results in 1 . For variability for recovery time, let 1 represent low variability, and 0 high variability. Thus, comparing alternatives $\mathrm{A}$ and $\mathrm{B}$ with regards to this attribute results in 1 . This procedure serves to reduce the number of parameters for the model prediction. (Details and all possible comparison values with respect to attributes and alternatives are listed in Appendix A.) Furthermore, these comparison values are weighted according to the DM's mean valence for each attribute. Assume two DMs, both with the same mean valences for cost (most important), intensity of pain, and recovery time (least important), but with different risk attitudes. In particular, let the mean valence (the weight) for the cost attribute be $\delta_{1}=.2$, for the intensity of pain attribute $\delta_{2}=.1$, and for variability of recovering time $\delta_{3}=.05$. The conflict parameter is set to $\gamma=.01$ or $\gamma=-.01$, depending on the type of conflict, the attention switching rate is $w_{i j}=.02$, and the decision boundary is $\theta=10$. Figure 2 shows the quantitative predictions of MDFT for this example. In particular, the stars and circles refer to the predictions of the risk-aversive and risk-seeking DMs, respectively.

The pattern indicates that predicted choice probabilities and decision times change in a systematic way, depending on the choice alternatives. Consider, for example, the choice pairs $A B$ and $C D$. The probabilities for choosing $\mathrm{A}$ over $\mathrm{B}$ and $\mathrm{C}$ over $\mathrm{D}$ are about the same. However, their corresponding decision times differ considerably. It takes longer to decide between $\mathrm{A}$ and $\mathrm{B}$ (both alternatives with high costs) than between $\mathrm{C}$ and $\mathrm{D}$ (both alternatives with low costs). That is, the avoidance conflict for an important attribute-that is, paying a lot of money-prolongs the decision time for making a decision between $\mathrm{A}$ and $\mathrm{B}$.

Moreover, the pattern of choice probability and decision time depends on the DM's risk attitude. For the riskseeking DM, B is preferred over A, and D is preferred over C. For the risk-aversive DM, the opposite is true. Now consider choice pairs AC (low variance) and BD (high variance). Here, the model predicts exactly opposite decision time patterns for the risk-seeking (circles) and the risk-aversive (stars) DMs. For the risk-seeking $\mathrm{DM}$, the decision time for $\mathrm{AC}$ is longer (avoidance) than for BD. The reverse pattern applies for the risk-aversive DM. That is, the model predicts different choice probabilities and decision time patterns, depending on the in- 

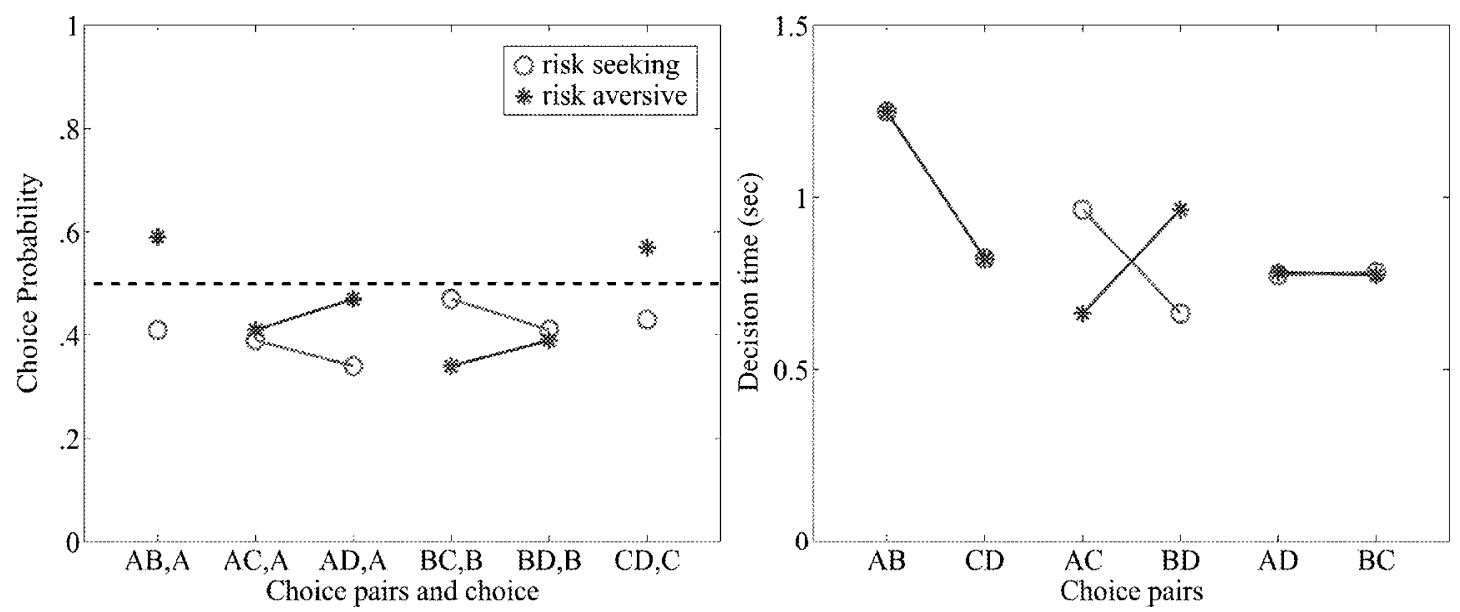

Figure 2. Predicted choice probabilities and decision times depending on risk attitude. AB,A indicates that choice pair AB was presented and A was chosen. The mean decision times are weighted mean decision times for choosing either of the alternatives.

dividual DM's valence for riskiness. The effect of the growth parameter $\gamma$ is an important feature of the model, and its representation of conflict types is characteristic for both DFT and MDFT. Without assuming a conflict parameter (i.e., $\gamma=0$ ), the decision times for choice pairs $\mathrm{AB}$ and $\mathrm{CD}$ and for choice pairs $\mathrm{AC}$ and $\mathrm{BD}$ are about the same. Note that the $\gamma$ in this example only changes its sign, depending on the type of conflict.

These quantitative predictions were derived by assuming specific parameters. However, MDFT makes more general predictions for the given attribute structure, as will be summarized next. In the following, choice probabilities are denoted as $\operatorname{Pr}(\mathrm{A}, \mathrm{B})$ for choosing $A$ over $B$. Mean decision times for the choice pairs are labeled $\mathrm{RT}_{\mathrm{AB}}$ for $\mathrm{A}$ and $\mathrm{B}$, and so on. Note that $\mathrm{RT}_{\mathrm{AB}}$ is the weighted mean decision time for choosing $\mathrm{A}$ over $\mathrm{B}$ and $\mathrm{B}$ over $\mathrm{A}$.

1. Risk Attitude. ${ }^{4}$

(a) Assuming that the DM is risk averse, then

$\operatorname{Pr}(\mathrm{A}, \mathrm{B})>.5$,

$\operatorname{Pr}(\mathrm{C}, \mathrm{D})>.5$,

$\operatorname{Pr}(\mathrm{A}, \mathrm{C}) \leq \operatorname{Pr}(\mathrm{A}, \mathrm{D})$,

$\operatorname{Pr}(\mathrm{B}, \mathrm{C}) \leq \operatorname{Pr}(\mathrm{B}, \mathrm{D})$

and the predicted decision time order is

$$
\mathrm{RT}_{\mathrm{AC}}<\mathrm{RT}_{\mathrm{BD}} \text {. }
$$

(b) If the DM is risk seeking, the inequalities of $1(a)$ are reversed.

2. Importance of attributes. ${ }^{5}$

(a) If the cost attribute is more important for a decision, then

$\operatorname{Pr}(\mathrm{A}, \mathrm{C})<.5$,

$\operatorname{Pr}(\mathrm{B}, \mathrm{D})<.5$,

and the predicted decision time order is

$\mathrm{RT}_{\mathrm{AB}}>\mathrm{RT}_{\mathrm{CD}}$. (b) If, however, the intensity of pain attribute is more important, the inequalities of $2(a)$ are reversed.

For the medical example above, the order relations between the probabilities and the decision times are illustrated in Figure 2, indicated by lines between them.

These predictions are tested in the following experiment that utilizes different attributes but possesses an attribute structure similar to the example.

\section{EXPERIMENT}

In order to induce conflict, decisions should have real consequences for the participant. Therefore, the choice alternatives are composed of three attributes: (1) amount of money to be won or lost, (2) intensity of an unpleasant sound, and (3) variability of the duration of that sound. In each trial, the participant may lose or win money and is forced to listen to noise of a particular intensity for a particular amount of time. Note that this attribute structure is similar to the medical example above, while allowing for real consequences.

Since the model is to be evaluated with individual decision-making behavior, estimation of choice probability and decision time requires that the same choice alternatives must repeatedly be presented to the participant. ${ }^{6}$

Table 2

Means of the Normal Distribution for the Three Attributes of the Four Alternatives

\begin{tabular}{crrrcc}
\hline & \multicolumn{3}{c}{ Money } & & \\
\cline { 2 - 4 } Alternative & \multicolumn{1}{c}{ I } & II & III & Intensity & Duration \\
\hline A & -1 & -2 & -1 & 75 & 15 \\
B & -1 & -2 & -1 & 75 & $15^{*}$ \\
C & 1 & 2 & 2 & 87 & 15 \\
D & 1 & 2 & 2 & 87 & $15^{*}$ \\
\hline
\end{tabular}

*Standard deviations of $\sigma=5$; for the remaining distributions, $\sigma=1.1$. 


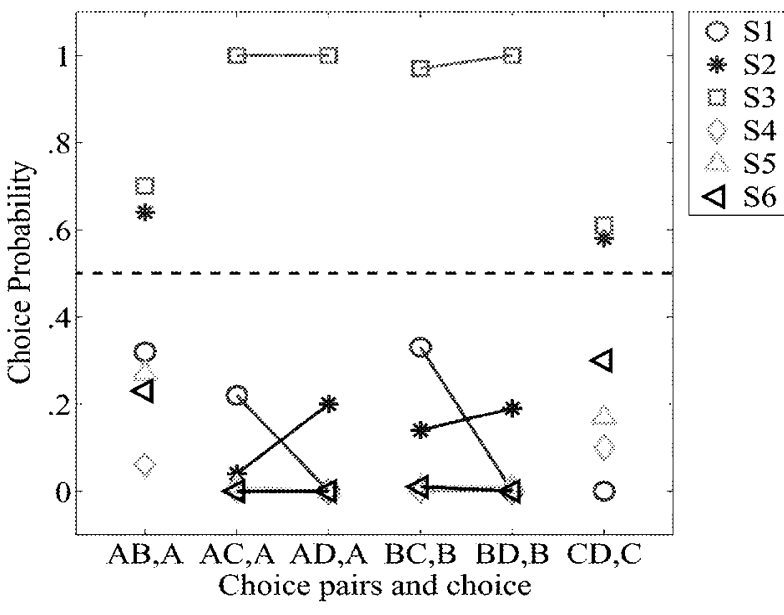

Figure 3. Choice probabilities for all choice pairs. Each choice probability was based on 90 observations.

In order to minimize the chance that the participants would simply remember their previous choices, the values of the choice alternatives were not identical but were drawn from distributions. The participants learned the distributions prior to the decision experiment by feedback (see below).

\section{Method}

Design. Four alternatives, labeled A, B, C, and D, all having the three attributes in common, were designed. The specific values of the attributes, drawn from truncated normal distributions and rounded to integer numbers, were money to be gained or lost (presented by integer numbers; each unit was worth 0.04 Deutsch Mark), intensities of a noise (presented by integer numbers $x$ in the range from 1 to 40; the actually delivered intensity, in decibels, was $x+55$ ), and duration of that noise (in seconds). The distributions of the attribute values differed with respect to mean and variance. In particular, A and B had the same negative expected values for money and the same mean intensity level $(75 \mathrm{~dB})$ for noise; $\mathrm{C}$ and $\mathrm{D}$ had the same positive expected values for money and the same mean intensity level $(87 \mathrm{~dB})$ for noise. The mean for duration was the same for all four alternatives $(15 \mathrm{sec})$, but the distributions differed with respect to variance - that is, for $\mathrm{A}$ and $\mathrm{C}$, the standard deviation was $\sigma=1.1 \mathrm{sec}$, whereas for $\mathrm{B}$ and $\mathrm{D}$, it was $\sigma=5 \mathrm{sec}$. Thus, $\mathrm{A}$ and $\mathrm{B}$ were identical with respect to the means but differed with respect to the variance of noise duration. The same holds for $\mathrm{C}$ and D. Table 2 shows the means of normal distributions from which the attribute values were drawn. The asterisk $(*)$ indicates distributions with standard deviations of $\sigma=5$, the remaining distributions having standard deviations of $\sigma=1.1$. Altogether, three different sets of parameters were designed, labeled Groups I, II, and III, respectively. They differed only according to the means for money. The resulting six choice pairs were labeled $\mathrm{AB}, \mathrm{AC}, \mathrm{AD}, \mathrm{BC}, \mathrm{BD}$, and $\mathrm{CD}$.

Participants. Three women (S1, S3, and S6) and three men (S2, S4, and S5) participated in this experiment. They were all associated with the Psychology Department of Oldenburg University. They were randomly assigned to Group I, II, or III. The participants received required course credits and/or 10 Deutsch Mark per session, plus the amount of money they won during each session.

Procedure. Each trial began with a display of two letters on a computer screen, representing the choice alternatives. The participant was asked to choose one of the two letters by pressing the respective button on a response box. The chosen letter appeared on the screen, and 2 sec later, feedback was delivered; the screen displayed the numerical values of the payoffs produced by each action of that trial. This feedback was followed by the actual delivery of the payoff produced by the letter chosen in that trial. The actual values of the payoffs and the probabilities produced by each action were learned through experience from trial to trial by feedback. The choice responses and decision times (in milliseconds) for each trial were recorded by the computer. The noises were presented binaurally over closed headphones; they were generated by a synthesizer and sampled by a sound card. Each group attended three sessions with 108 trials each for training and five experimental sessions with 108 trials each. Thus, for six choice pairs, 540 experimental trials were recorded for each participant.

\section{Results}

Figure 3 shows the choice probabilities for all 6 participants. The patterns indicate the more desirable attribute and the risk attitude for each participant. None of the participants made an exact tradeoff between money to be won and intensity level [i.e., $\operatorname{Pr}(\mathrm{A}, \mathrm{C}) \neq .5$ and $\operatorname{Pr}(\mathrm{B}, \mathrm{D}) \neq .5]$, nor were they indifferent to variability in duration [i.e., $\operatorname{Pr}(\mathrm{A}, \mathrm{B}) \neq .5$ and $\operatorname{Pr}(\mathrm{C}, \mathrm{D}) \neq .5$ ].

Most notably, 2 participants, S2 and S3, differed from the rest.

First, probabilities for choosing A (or B) $(-\$$, soft) over C (or D) ( $\$$, loud) were considered as an indicators for the DM's importance of attributes. For $\operatorname{Pr}(\mathrm{A}, \mathrm{C})<$ .5 and $\operatorname{Pr}(\mathrm{B}, \mathrm{D})<.5$, it was inferred that money was more important than noise, whereas the opposite applied when $\operatorname{Pr}(\mathrm{A}, \mathrm{C})>.5$ and $\operatorname{Pr}(\mathrm{B}, \mathrm{D})>.5$. Thus, for 5 out of 6 participants, the most important attribute in deciding for an alternative was money-that is, they were more likely to choose C (or D) over A (or B). For these 5 participants, the mean decision time was predicted to be larger when the participant chose between A and B (the alternatives with negative monetary expectations) than when the participant chose between $\mathrm{C}$ and $\mathrm{D}$ (the alternatives with

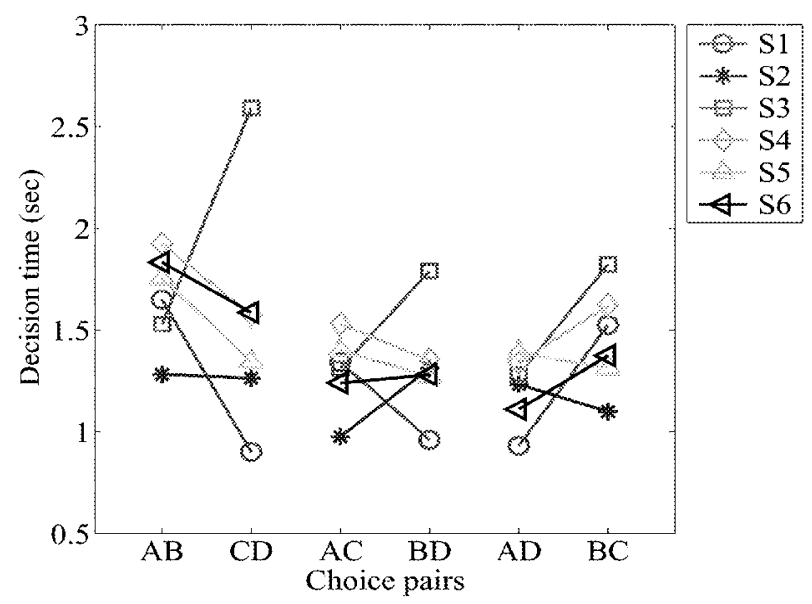

Figure 4. Mean decision times (in seconds). Each decision time was based on 90 observations. Decision times for AB and CD were related to conflict evoked by attribute values; decision times for $\mathrm{AC}$ and $\mathrm{BD}$ were related to conflict evoked by risk attitude; decision times $\mathrm{AD}$ and $\mathrm{BC}$ are additional observations. 

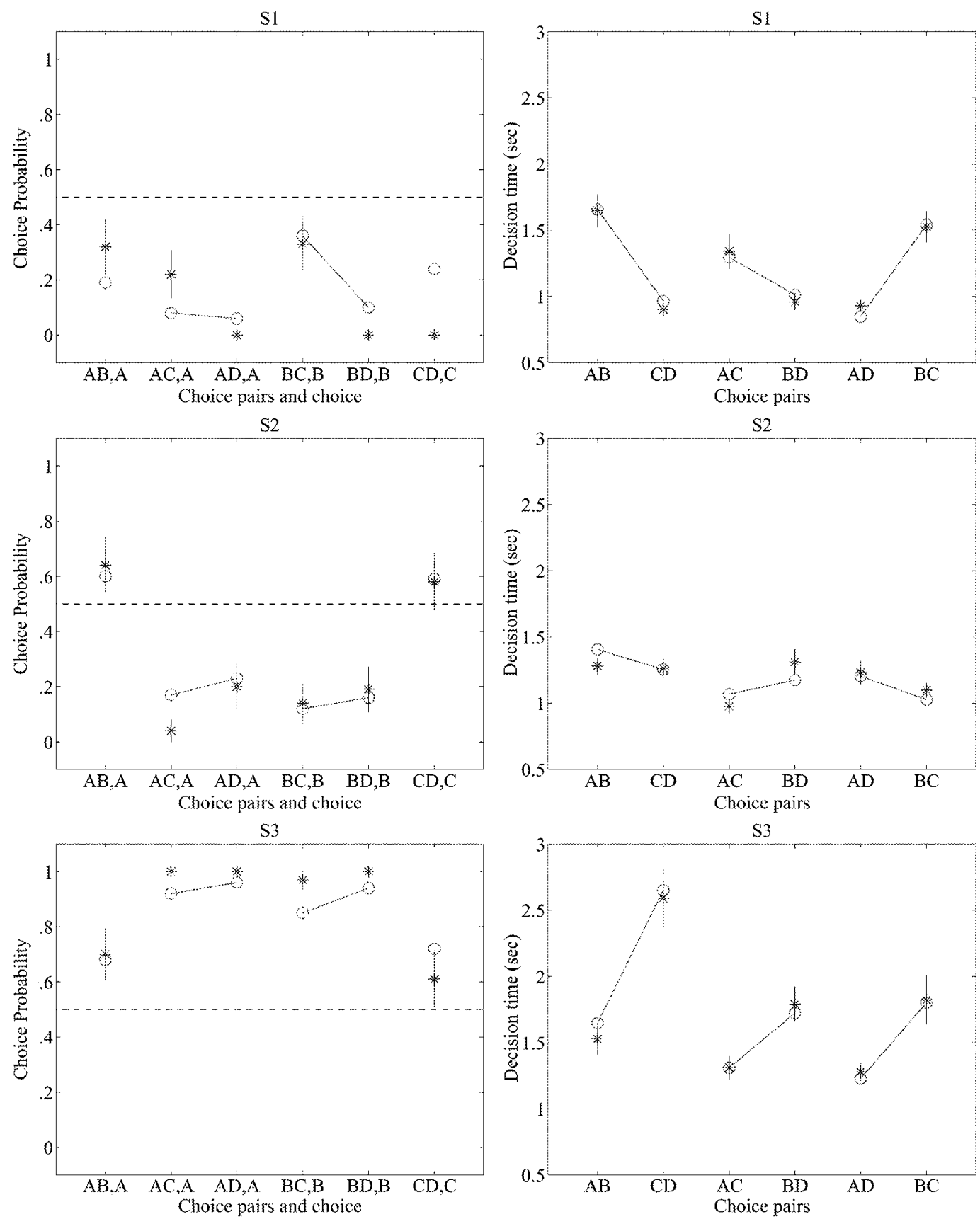

Figure 5. Observed (stars) and predicted (circles) choice probabilities (left panels) and decision times (right panels) for the six choice pairs.

positive monetary expectations). For 1 participant (S3), the alternatives with the highest intensity were the least desirable. For her, the mean decision time for choosing between $\mathrm{A}$ and $\mathrm{B}$ should have been less than the mean decision time for choosing between $\mathrm{C}$ and $\mathrm{D}$.

Second, probabilities for choosing A (or C) (low variability) over B (or D) (high variability) were considered as one indicator for the participant's risk attitude. For
$\operatorname{Pr}(\mathrm{A}, \mathrm{B})<.5$ and $\operatorname{Pr}(\mathrm{C}, \mathrm{D})<.5$, it was inferred that high variability was preferred to low variability. In addition, this attitude to risk was also inferred by comparing the probabilities of choosing $\mathrm{A}$ ( or B) over $\mathrm{C}$ with the probabilities of choosing A (or B) over D. Four out of 6 participants chose the alternatives with the higher variability in duration over the less variable alternatives. For them, the mean decision time for choosing between $\mathrm{A}$ and $\mathrm{C}$ 
S4

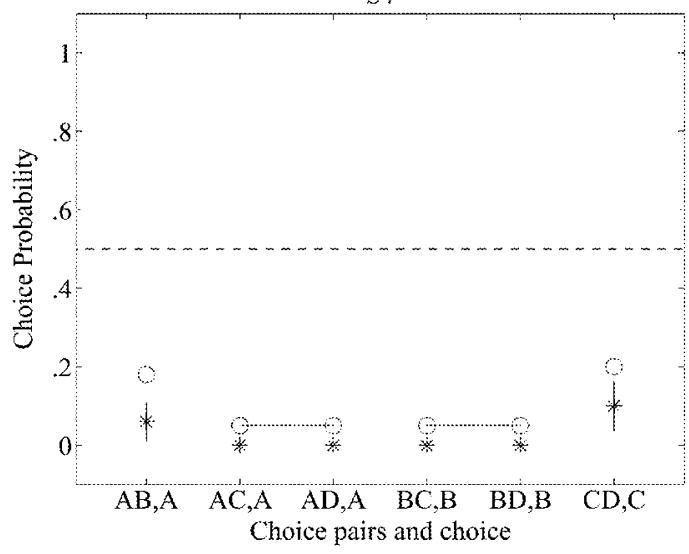

S5

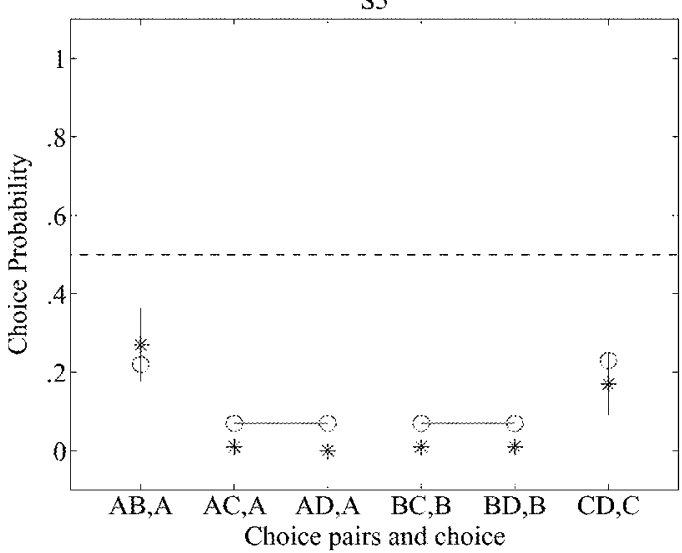

S6

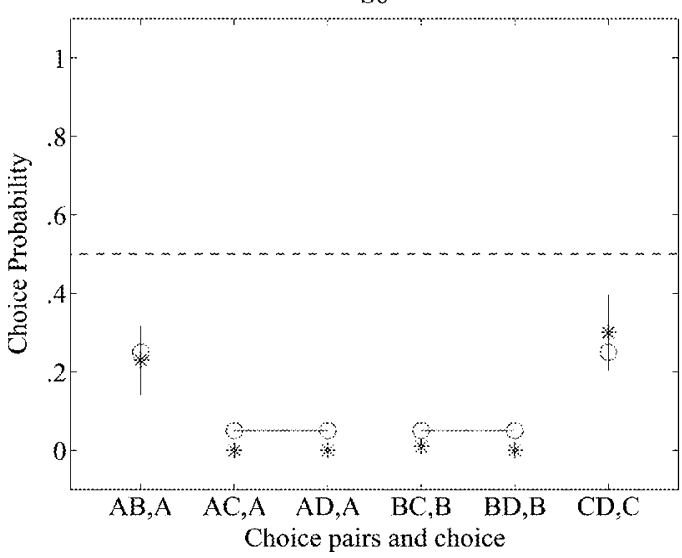

S4

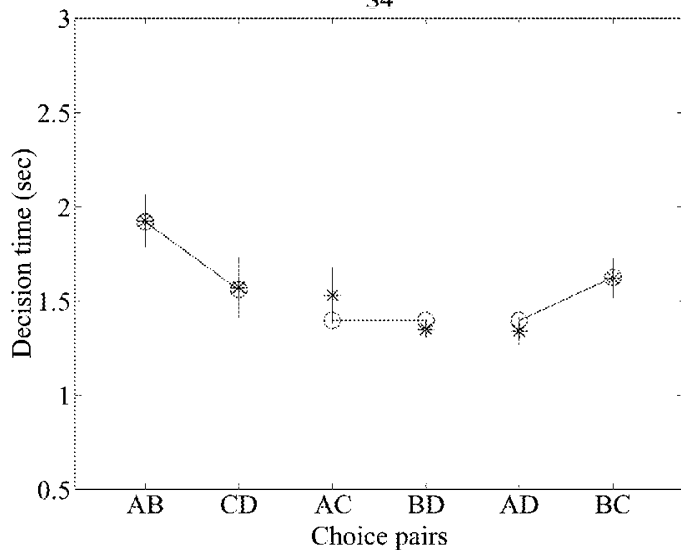

S5

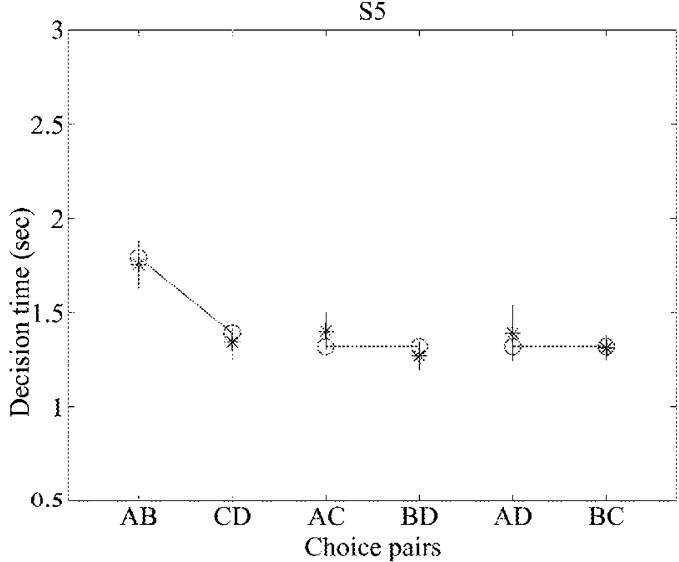

S6

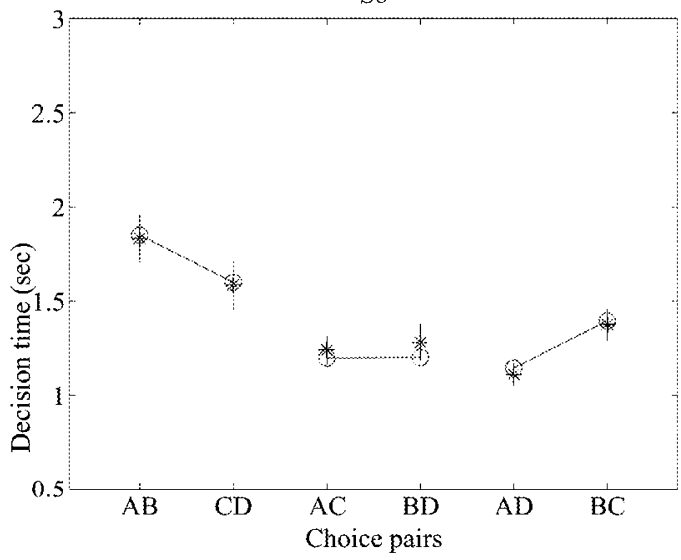

Figure 5 (Continued).

should be longer than that for choosing between $\mathrm{B}$ and $\mathrm{D}$. For the remaining 2 participants, $\mathrm{S} 2$ and S3, the mean decision time when choosing between $\mathrm{A}$ and $\mathrm{C}$ should have been less than when choosing between $\mathrm{B}$ and $\mathrm{D}$.

To summarize, according to the basic hypothesis, patterns of choice probability predict decision times. Therefore, for Participants S1, S4, S5, and S6, the predicted decision time order was $\mathrm{RT}_{\mathrm{AB}}>\mathrm{RT}_{\mathrm{CD}}$ ( since money was more important than noise and, therefore, losing money [both A and $\mathrm{B}$ ] evoked an avoidance conflict, prolonging the decision time for that choice pair) and $\mathrm{RT}_{\mathrm{AC}}>\mathrm{RT}_{\mathrm{BD}}$ (since high risk was preferred to low risk [both $\mathrm{A}$ and $\mathrm{C}$ ] and, therefore, prolonged the decision time for that choice pair). For Participant S3, the opposite order was predicted - that is, $\mathrm{RT}_{\mathrm{AB}}<\mathrm{RT}_{\mathrm{CD}}$ ( since noise was more important than money and, therefore, listening to loud 
noises [both $\mathrm{C}$ and $\mathrm{D}$ ] evoked an avoidance conflict, prolonging the decision time for that choice pair) and $\mathrm{RT}_{\mathrm{AC}}<\mathrm{RT}_{\mathrm{BD}}$ (since low risk was preferred to high risk [alternatives $B$ and D] ). For Participant S2, the predicted order was $\mathrm{RT}_{\mathrm{AB}}>\mathrm{RT}_{\mathrm{CD}}$ (since money was more important than noise) and $\mathrm{RT}_{\mathrm{AC}}<\mathrm{RT}_{\mathrm{BD}}$ (since low risk was preferred to high risk). Figure 4 shows the observed decision time for each condition and participant.

With respect to the choice pairs $A B$ and $C D$, the predicted order could be observed in all 6 participants; the mean difference was significant $(p<.01)$ for all the participants but S2 (for test results, see Appendix B). According to the hypothesis, conflict strength induced by undesirable values (money to lose, very high intensity of noise) can be measured by decision time. Indeed, the longest decision times could be observed for the most aversive decisions (avoidance-avoidance conflict). Moreover, the results suggest that conflict induced by variability (uncertainty) is also measurable by decision time (comparing choice pairs AC and BD). MDFT predicts the order of decision time qualitatively, depending on the individual choice probability pattern of a DM. That it can also be fitted quantitatively to the data is shown in the following section. In addition, it makes quantitative predictions for the choice pairs $\mathrm{AD}$ and $\mathrm{BC}$ that cannot be made qualitatively.

\section{FITTING MDFT TO THE DATA}

To restrict the model and, thereby, impose a strong restriction on the data, MDFT is fitted to the observed complex choice patterns under the following assumptions. (1) To account for the finding that effects of sound intensity and duration are additive (Schreiber \& Kahneman, 2000 ), intensity and duration are not treated as separate attributes but are combined, reducing the number of attributes to two (see Appendix A). (2) Decision criteria are symmetric - that is, there is no a priori bias for any alternative. (3) The rate of switching from one attribute to another is the same for all attributes and is time invariant. (4) The parameters for conflict, evoked by attribute values and/or by risk attitude are the same, although the sign may be different. (5) To further reduce the number of parameters of the model, the parameters of the distributions for generating the alternatives enter into the model parameters. The mean values stated in Table 2 are mapped to 0 and 1 according to magnitude: For each attribute, 0 represents the smaller value, and 1 represents the larger value, similar to the values in Table 1. Two attributes are compared by determining the difference between their values, similar to the procedure used to construct Table A1 in Appendix A.

Altogether, for each participant there are six model parameters to be estimated from the data (12 independent observations-i.e., six relative frequencies and six mean decision times): $\delta_{1}$, to determine the mean valence when two alternatives are compared with respect to attribute money; $\delta_{2}$, to determine the mean valence when two alternatives are compared with respect to attribute intensity; $\delta_{3}$, to determine the mean valence when two alternatives are compared with respect to variance of duration; $\gamma$, to determine the growth or decay of the process, depending on the conflict situation; $\theta$, the decision criterion; and $w_{i j}=w_{j i}$, the attention switching rate, for all attributes $i, j \in\{1,2\}, i \neq j$.

These parameters were estimated by minimizing the sum of squared deviations of the observed and predicted choice probabilities and decision times, using the FMINSEARCH routine of MATLAB.

The observed choice probabilities and decision times and the predictions of MDFT for all 6 participants are shown in Figure 5. The model describes the decision times emphasized in this study remarkably well for all 6 participants. MDFT accounts in quantitative detail for the complete set of decision times. Moreover, MDFT captures the pattern of observed choice probabilities, with an exception involving S $1 .{ }^{7}$ Furthermore, this intricate set of predictions could not have been made without the model.

\section{CONCLUSION}

The primary purpose of this study was to investigate the usefulness of decision time as a measure of conflict strength in a risky decision-making situation. The results of the present experiment suggest that decision time can be considered as a measure of conflict induced by both the desirability/undesirability of values and the variability of outcomes for decision making under uncertainty. MDFT accounted for the empirical findings by actually predicting a decision time pattern depending on conflict types. Note that without the theory, the decision time patterns could not be predicted simply on the basis of choice probabilities. That is, the same choice probabilities may be associated with different decision times, and vice versa. Furthermore, the model is able to account for individual differences, which provides a strong test of the model. Four different preference patterns were predicted with respect to risk attitude (risk seeking/risk averse) and to attribute preference (money/noise). Three of these patterns were observed in the data: For 5 participants, the amount of money to win or to lose was the most important attribute in deciding for an alternative; 4 of these were risk seeking, 1 risk avoiding. For 1 participant, the intensity of noise was the most important attribute in deciding for an alternative, and she was risk avoiding. Even with strong simplifying restrictions imposed on the model, MDFT was able to account for these patterns.

The empirical results support Tversky and Shafir (1992), who suggested that deferring a decision may be considered as one indicator of conflict. Furthermore, MDFT gives a strong theoretical foundation for their ideas in the context of Lewin's psychodynamic approach.

So far, MDFT seems to be the only model to account for the complex pattern of choices and response times of 
decision making in conflict situations with multiattribute choice alternatives at the individual level, both qualitatively and quantitatively.

\section{REFERENCES}

Berlyne, D. E. (1957). Conflict and choice time. British Journal of Psychology, 48, 106-118.

Böckenholt, U., Albert, D., Aschenbrenner, M., \& SchmalHOFER, F. (1991). The effects of attractiveness, dominance, and attribute differences on information acquisition in multiattribute binary choice. Organizational Behavior \& Human Decision Processes, 49, 258-281.

BuSEMEYER, J. R. (1985). Decision making under uncertainty: A comparison of simple scalability, fixed-sample, and sequential-sampling models. Journal of Experimental Psychology: Learning, Memory, \& Cognition, 11, 538-564.

Busemeyer, J. R., \& Diederich, A. (2002). Survey of decision field theory. Mathematical Social Sciences, 43, 345-370.

Busemeyer, J. R., \& Townsend, J. T. (1992). Fundamental derivations from decision field theory. Mathematical Social Sciences, 23, 255282.

Busemeyer, J. R., \& Townsend, J. T. (1993). Decision field theory: A dynamic-cognitive approach to decision-making in an uncertain environment. Psychological Review, 100, 432-459.

Coombs, C. H., \& Avrunin, G. S. (1988). The structure of conflict. Hillsdale, NJ: Erlbaum.

DASHIELl, J. F. (1937). Affective value-distances as a determinant of esthetic judgment-times. American Journal of Psychology, 50, 5767.

DiEDERICH, A. (1995). A dynamic model for multi-attributive decision problems. In J. P. Caverni, M. Bar-Hillel, F. H. Barron, \& H. Jungermann (Eds.), Contributions to decision making (Vol. I, pp. 175-191). Amsterdam: Elsevier.

Diederich, A. (1996). Multi-attribute dynamic decision model: A cognitive dynamic approach for multiattribute binary choice tasks. Unpublished Habilitationsschrift, Universität Oldenburg.

DiEDERICH, A. (1997). Dynamic stochastic models for decision making with time constraints. Journal of Mathematical Psychology, 41, 260274.

Diederich, A., \& Busemeyer, J. R. (1999). Conflict and the stochastic dominance principle of decision making. Psychological Science, 10, 353-359.

HANSEn, F. (1972). Consumer choice behavior: A cognitive theory. New York: Free Press

Hogarth, R. M. (1975). Decision time as a function of task complexity. In D. Wendt \& C. Vlek (Eds.), Utility, probability, and human decision making (pp. 321-338). Dordrecht: Reidel.

Houston, D. A., Sherman, S. J., \& Baker, S. M. (1991). Feature matching, unique features, and the dynamics of the choice process: Predecision conflict and postdecision satisfaction. Journal of Experimental Social Psychology, 27, 411-430.

Hull, C. L. (1932). The goal gradient hypothesis and maze learning. Psychological Review, 39, 25-43.

JANIS, I. L., \& MANN, L. (1977). A psychological analysis of conflict, choice, and commitment. New York: Free Press.

LEWIN, K. (1951). Field theory in social science. Selected theoretical papers, edited by D. Cartwright. New York: Harper \& Brothers.

LewIN, K. (1964). Die psychologische Situation bei Lohn und Strafe [The psychological situation of reward and punishment]. Darmstadt: Wissenschaftliche Buchgesellschaft. (Original work published 1931)

Luce, M. F., Bettman, J. R., \& Payne, J. W. (1997). Choice process- ing in emotionally difficult decisions. Journal of Experimental Psychology: Learning, Memory, \& Cognition, 23, 384-405.

Mellers, B., Schwartz, A., Ho, K., \& Ritov, I. (1997). Decision affect theory: How we feel about risky options. Psychological Science, 8, 423-429.

Miller, N. E. (1944). Experimental studies of conflict. In J. M. Hunt (Ed.), Personality and the behavior disorders (Vol. 1, pp. 431-465). New York: Ronald Press.

Payne, J. W., Bettman, J. R., \& Schkade, D. A. (1999). Measuring constructed preferences: Towards a building code. Journal of Risk \& Uncertainty, 19, 243-270.

Roe, R. M., Busemeyer, J. R., \& Townsend, J. T. (2001). Multialternative decision field theory: A dynamic connectionist model of decision making. Psychological Review, 108, 370-392.

Schreiber, C. A., \& Kahneman, D. (2000). Determinants of the remembered utility of aversive sounds. Journal of Experimental Psychology: General, 129, 27-42.

Townsend, J. T., \& Busemeyer, J. R. (1989). Approach-avoidance: Return to dynamic decision behavior. In C. Izawa (Ed.), Current issues in cognitive processes: The Tulane Flowerree Symposium on Cognition (pp. 107-133). Hillsdale, NJ: Erlbaum.

Tversky, A., \& Shafir, E. (1992). Choice under conflict: The dynamics of deferred decision. Psychological Science, 3, 358-361.

\section{NOTES}

1. The choice alternatives have desirable and undesirable attributes and, therefore, evoke an approach-avoidance conflict. However, if the attributes that evoke an approach conflict are more important to the DM than the attributes that evoke an avoidance conflict, the conflict will simply be called approach conflict. A pure approach conflict requires the values of all attributes to be desirable for the DM. The same holds for avoidance conflicts.

2 . Note that by switching from one attribute to another within one trial, the entire process is not time homogeneous any more. That is, the drift changes with time within one trial. Setting $w_{i j}=0$ (no switching) and assuming a noisy average of the mean valences of each attribute results in a drift that does not change within one trial. On the other hand, for MDFT, the drift may change from positive to negative within one trial.

3. Multiattribute decision field theory was first introduced under the label MDFT in Diederich (1995; see also Diederich \& Busemeyer, 1999). However, there should be no confusion when considered in the respective context.

4. Assuming that the DM is indifferent to various degrees of variability (i.e., ignoring the variability of recovery time attribute), then $\operatorname{Pr}(\mathrm{A}, \mathrm{B})=\operatorname{Pr}(\mathrm{B}, \mathrm{A})=\operatorname{Pr}(\mathrm{C}, \mathrm{D})=\operatorname{Pr}(\mathrm{D}, \mathrm{C})=.5$, and $\operatorname{Pr}(\mathrm{A}, \mathrm{C})=\operatorname{Pr}(\mathrm{A}, \mathrm{D})$, and $\operatorname{Pr}(\mathrm{B}, \mathrm{C})=\operatorname{Pr}(\mathrm{B}, \mathrm{D})$. The predicted decision time relation is $\mathrm{RT}_{\mathrm{AC}}=$ $\mathrm{RT}_{\mathrm{BD}}$.

5. Assuming that the cost and intensity of pain attributes are equally important to the $\mathrm{DM}$ to determine a decision, then $\operatorname{Pr}(\mathrm{A}, \mathrm{C})=\operatorname{Pr}(\mathrm{C}, \mathrm{A})=$ $\operatorname{Pr}(\mathrm{B}, \mathrm{D})=\operatorname{Pr}(\mathrm{D}, \mathrm{B})=.5$, and the predicted decision time relation is $\mathrm{RT}_{\mathrm{AB}}=\mathrm{RT}_{\mathrm{CD}}$.

6 . This method, commonly used in psychophysics, has recently been proposed to be applied in decision-making studies (e.g., Payne, Bettman, \& Schkade, 1999).

7. For S1, MDFT cannot account for the observed choice probability for choosing $\mathrm{C}$ over $\mathrm{D}$ without making further assumptions. MDFT predicts about the same probabilities for choosing A over B and C over D, since $\mathrm{A}$ and $\mathrm{C}$ (B and $\mathrm{D}$ ) are similar with respect to attribute variability of duration. However, there might be an interaction between duration and intensity of noise that is not captured simply by adding intensity and duration. Obviously, the assumed structure was too simple to account for the observed pattern. 


\section{APPENDIX A}

For the example presented in Table 1, the drift coefficients were determined by weighing the difference values of two attribute comparisons, shown in Table A1. Low variability of variability of recovery time is labeled by 1 ; high variability by 0 . For each choice pair, indexed $i, i=1,2, \ldots, 6$, the drift rate for costs was set to $\mu_{i 1}(x)=\delta_{1} \cdot y_{i 1}-\gamma \cdot y_{i 4} \cdot x$; for pain, it was set to $\mu_{i 2}(x)=\delta_{2} \cdot y_{i 2}$; and for recovering, it was set to $\mu_{i 3}(x)=$ $-\delta_{3} \cdot y_{i 3}-\gamma \cdot y_{i 4} \cdot x$ for the risk-seeking DM and to $\mu_{i 3}(x)=\delta_{3}$. $y_{3 j}+\gamma \cdot y_{i 4} \cdot x$ for the risk-aversive DM. The sign of $\delta_{3}$ and $\gamma$ determines the risk attitude.

Table A1

Comparison (Difference) Values for All Choice Pairs, $i=1,2,3$, 4, 5, 6, With Respect to Attributes and Conflict Structure

\begin{tabular}{|c|c|c|c|c|c|}
\hline \multirow[b]{2}{*}{$\begin{array}{l}\text { Choice Pairs } \\
\text { and Index }(i)\end{array}$} & \multicolumn{3}{|c|}{ Comparison Values for } & \multicolumn{2}{|c|}{ Conflict Evoked by } \\
\hline & $\begin{array}{l}\text { Cost } \\
\left(y_{i 1}\right)\end{array}$ & $\begin{array}{l}\text { Pain } \\
\left(y_{i 2}\right)\end{array}$ & $\begin{array}{c}\text { Recovery Time } \\
\left(y_{i 3}\right)\end{array}$ & $\begin{array}{c}\text { Values } \\
\left(y_{i 4}\right)\end{array}$ & $\begin{array}{l}\text { Risk } \\
\left(y_{i 5}\right)\end{array}$ \\
\hline $\mathrm{AB}, 1$ & 0 & 0 & 1 & 1 & 0 \\
\hline $\mathrm{AC}, 2$ & -1 & 1 & 0 & 0 & 1 \\
\hline $\mathrm{AD}, 3$ & -1 & 1 & 1 & 0 & 0 \\
\hline $\mathrm{BC}, 4$ & -1 & 1 & -1 & 0 & 0 \\
\hline $\mathrm{BD}, 5$ & -1 & 1 & 0 & 0 & -1 \\
\hline $\mathrm{CD}, 6$ & 0 & 0 & 1 & -1 & 0 \\
\hline
\end{tabular}

Note-The parameters (weights) were set to $\delta_{1}=.2, \delta_{2}=.1, \delta_{3}=.05$, and $\gamma=.02$. The switching rate (attention shift) was set to .01 , and the decision criterion to 10 .

\section{APPENDIX B}

The drift coefficients for fitting the model to the data are

$$
\mu_{i 1}(x)=\delta_{1} \cdot y_{i 1}-\gamma \cdot y_{i 4} \cdot x
$$

for considering money and

$$
\mu_{i 2}(x)=\left(\delta_{2} \cdot y_{i 2}+\delta_{2} \cdot y_{i 2}\right)-\gamma \cdot y_{i 5} \cdot x
$$

for the intensity/duration combination.

Table B1

Model Parameters for Each Participant

\begin{tabular}{crrrrrr}
\hline Participant & \multicolumn{1}{c}{$\delta_{1}$} & \multicolumn{1}{c}{$\delta_{2}$} & \multicolumn{1}{c}{$\delta_{3}$} & \multicolumn{1}{c}{$\gamma$} & $\theta$ & $w_{i j}$ \\
\hline S1 & .064 & 0.139 & -0.153 & .009 & 13 & .003 \\
S2 & .069 & 0.076 & 0.036 & .003 & 11 & .102 \\
S3 & -.076 & -0.103 & 0.060 & -.007 & 15 & .003 \\
S4 & .051 & 2.280 & -1.917 & .001 & 20 & .001 \\
S5 & .047 & 2.154 & -1.140 & .002 & 18 & .001 \\
S6 & .065 & 0.557 & -0.314 & .000 & 17 & .000 \\
\hline
\end{tabular}

(Manuscript received April 17, 2001;

revision accepted for publication February 22, 2002.) 